\title{
Revisiting the Strait of Messina: The Balance Between Optimal Oncologic Outcomes and Complications From Surgery
}

\author{
Keith A. Delman, MD and Michael C. Lowe, MD \\ Division of Surgical Oncology, Department of Surgery, Winship Cancer Institute, Emory University, Atlanta, GA
}

\begin{abstract}
In 2011 Vern Sondak and Amod Sarnaik penned an editorial accompanying the first report of minimally invasive inguinal lymphadenectomy aptly referencing Scylla and Charybdis of Greek mythology. We revisit that initial editorial with a commentary on this issue's call to arms by Faut and colleagues.
\end{abstract}

The discourse regarding the balance of oncologic benefit and complication profile for lymphadenectomy has been front and center in both breast cancer and melanoma for well over a decade. ${ }^{1-9}$ arguably, inguinal lymphadenectomy has been a lightning rod for this debate because of the high morbidity that has repeatedly been reported from this procedure. The authors of this editorial admittedly have contributed to the ongoing rhetoric by developing and reporting a novel approach to inguinal lymphadenectomy. ${ }^{3,7}$ Combined with ongoing investigation into the role and utility of completion lymphadenectomy, this has led the melanoma community aggressively to revisit groin dissection in the therapeutic armamentarium of the melanoma surgeon.

In this issue of Annals of Surgical Oncology, Faut et al. analyze the impact of changes in perioperative care on the outcomes from inguinal lymphadenectomy. They grouped their analysis by three major changes related to mobility, splinting, and duration of hospitalization. Importantly, they meticulously analyzed the data for incidence of complications and included patients who underwent both superficial and combined superficial and deep lymphadenectomies. One could argue that these two populations are not the same and so inclusion of both in this manuscript weakens its conclusions, but nonetheless,

\section{(C) Society of Surgical Oncology 2016}

First Received: 16 August 2016; Published Online: 12 September 2016

K. A. Delman, MD

e-mail: kdelman@emory.edu the authors draw our attention to the challenge of surgery in this area of the body. While this group is trained and does use minimally invasive lymphadenectomy, the present report does not investigate the impact of that procedure on outcomes; all patients in the study underwent open inguinal lymphadenectomy.

Recent series (including the Faut series in this issue) estimating the morbidity from inguinal lymphadenectomy have been more comprehensive in reporting adverse outcomes than historical series. Modern data report complication rates ranging from 19-77\%, with the majority observing an estimated $50 \%$ of patients with a wound complication. ${ }^{3,5,10-15}$ This type of meticulous analysis has led to considerable discussion in the melanoma community about the true accuracy of reported complication rates after inguinal lymphadenectomy. While this debate may never be put to rest, it can be argued that it is irrelevant, because there is no debate that the rate of complications is significant. If one is to accept that the complication rate from this procedure is simply "too high" (whether that number is 20 or $50 \%$ ), then the discussion about reducing morbidity is valid and the call to arms of the group from Groningen is worthy of acknowledgement.

Including the current article, a number of modifications to surgical technique have been advocated to reduce morbidity from inguinal lymphadenectomy, and while minimally invasive surgery may be the most impactful, there are still significant complications even with this approach. ${ }^{3-9}$ Arguably, the optimal way to reduce complications is to avoid surgery altogether. Two trials, the Multicenter Selective Lymphadenectomy Trial-II and the EORTC's MiniTub registry trial, prospectively are analyzing the outcome of patients who do not undergo completion lymphadenectomy after a positive sentinel lymph node biopsy (SLNB). The melanoma community eagerly awaits the results of these trials, especially as recent data imply that some patients may avoid completion lymphadenectomy without adversely impacting overall 
survival. In 2015, the German Dermatologic Cooperative Oncology Group (DECOG) presented the early results of a trial randomizing 483 patients to completion lymphadenectomy or observation alone after positive SLNB from melanoma. ${ }^{16}$ The primary endpoint of this trial was distant metastasis-free survival, although additional endpoints were analyzed. Median follow-up in this trial was 3 years, and while regional recurrence was higher, melanoma-specific survival and distant metastasis-free survival were statistically equivalent. DECOG should be recognized for the successful completion of this trial; however, the results are to be interpreted with considerable caution. In patients with low-volume disease, as those included in the trial would be considered, the pattern of disease recurrence should consider the potential for an extended period of late recurrence. Results before 5 years, and quite arguably 10 years (as is incorporated into the design of the MSLT-II trial), should be considered premature and should not be independently acted upon.

While these data are premature, they open the door for a discussion about obviating completion lymphadenectomy in patients at high risk for complications. Furthermore, they provide greater equipoise for MSLT-II and Minitub. Importantly, in the context of the current article in this issue of Annals of Surgical Oncology, the DECOG trial further fuels the debate about the role of potentially eliminating a high-risk surgical procedure in patients who may, in fact, not benefit from it.

Over the past decade, remarkable improvements have been made in the understanding of melanoma. This progress has led to the approval and therapeutic use of a multitude of new agents leading to unprecedented transformations in care that have never occurred previously in melanoma. While many may view the introduction and success of these agents as an opportune time to reduce the role of surgery, it could be considered that instead, it is a time to restructure the role of surgery, even in a more aggressive context. In patients with successful options for systemic control, locoregional control may be even more important than in the past. One may argue that aggressive metastasectomy may see an increased role in patients who have successfully responded to systemic therapy. Regardless of one's perspective about the intersection of systemic therapy and surgery, the advent of these advances, combined with the results of recent studies analyzing the role of completion lymphadenectomy should prompt all clinicians to give pause and reconsider our approach to the melanoma patient.

In this light, it is conceivable that the approach to reduce complications from inguinal lymphadenectomy has been incorrect. While a multitude of experts have spent years trying to reduce the complications from a high-risk procedure by addressing the technical aspects of the procedure, it seems that the time is right to build on our growing understanding of the biology of this disease and also upon the data that show that SLNB may be therapeutic (or, dare it be said, "curative"?) in selected patients (conceptually it is provocative to consider that SLNB may be therapeutic if we are to accept that completion lymphadenectomy is not needed in selected patients). It is a time when we should come together as melanoma clinicians and characterize patients better than we have before. Attention should be focused on selecting patients who need and will benefit from new systemic therapies, those who will need and benefit from completion lymphadenectomy, and those who will not require either systemic therapy or completion lymphadenectomy. No medical or surgical intervention is without risk. Faut and colleagues are correct; morbidity is unacceptable and is yet to be mitigated for inguinal lymphadenectomy. We should focus on the entire spectrum of treatment: optimize selection of patients for all therapeutic options and avoid the temptation to so intensely focus our attention on one aspect of the therapeutic armentatrium over another.

\section{DISCLOSURES None.}

\section{REFERENCES}

1. Giuliano AE, Hunt KK, Ballman KV, et al. Axillary dissection vs no axillary dissection in women with invasive breast cancer and sentinel node metastasis: a randomized clinical trial. JAMA. 2011;305(6):569-75.

2. Olson JA Jr, McCall LM, Beitsch P, et al. Impact of immediate versus delayed axillary node dissection on surgical outcomes in breast cancer patients with positive sentinel nodes: results from American College of Surgeons Oncology Group Trials Z0010 and Z0011. J Clin Oncol. 2008;26(21):3530-5.

3. Master VA, Jafri SM, Moses KA, et al. Minimally invasive inguinal lymphadenectomy via endoscopic groin dissection: comprehensive assessment of immediate and long-term complications. J Urol. 2012;188(4):1176-80.

4. Martin BM, Master VA, Delman KA. Minimizing morbidity in melanoma surgery. Oncology. 2013;27(10):1016-20.

5. Buda A, Ghelardi A, Fruscio R, et al. The contribution of a collagen-fibrin patch (Tachosil) to prevent the postoperative lymphatic complications after groin lymphadenectomy: a double institution observational study. Eur J Obstet Gynecol Reprod Biol. 2016;197:156-8.

6. Matthey-Gié ML, Gié O, Deretti S, Demartines N, Matter M. Prospective randomized study to compare lymphocele and lymphorrhea control following inguinal and axillary therapeutic lymph node dissection with or without the use of an ultrasonic scalpel. Ann Surg Oncol. 2016;23(5):1716-20.

7. Delman KA, Kooby DA, Rizzo M, Ogan K, Master V. Initial experience with videoscopic inguinal lymphadenectomy. Ann Surg Oncol. 2011;18(4):977-82.

8. Spillane AJ, Tucker M, Pasquali S. A pilot study reporting outcomes for melanoma patients of a minimal access ilio-inguinal 
dissection technique based on two incisions. Ann Surg Oncol. 2011;18(4):970-6.

9. Sarnaik AA, Puleo CA, Zager JS, Sondak VK. Limiting the morbidity of inguinal lymphadenectomy for metastatic melanoma. Cancer Control. 2009;16(3):240-7.

10. Stuiver MM, Westerduin E, Ter Meulen S, et al. Surgical wound complications after groin dissection in melanoma patients: a historical cohort study and risk factor analysis. Eur J Surg Oncol. 2014;40(10):1284-90.

11. Sabel MS, Griffith KA, Arora A, et al. Inguinal node dissection for melanoma in the era of sentinel node biopsy. Surgery. 2007;141(6):728-35.

12. Serpel JW, Carne PW, Bailey M. Radical lymph node dissection for melanoma. ANZ J Surg. 2003;73(5):294-9.

13. Nelson BA, Cookson MS, Smith JA Jr, Chang SS. Complications of inguinal and pelvic lymphadenectomy for squamous cell carcinoma of the penis: a contemporary series. J Urol. 2004; 172(2):494-7.

14. Poos HP, Kruijff S, Bastiaannet E, van Ginkel RJ, Hoekstra HJ. Therapeutic groin dissection for melanoma: risk factors for short term morbidity. Eur J Surg Oncol. 2009;35(8):877-83.

15. Chang SB, Askew RL, Xing Y, et al. Prospective assessment of postoperative complications and associated costs following inguinal lymph node dissection (ILND) in melanoma patients. Ann Surg Oncol. 2010;17(10):2764-72.

16. Leiter U, Stadler R, Mauch C, et al. Survival of SLNB-positive melanoma patients with and without complete lymph node dissection: a multicenter, randomized DECOG trial. 2015 ASCO Annual Meeting (May 29_June 2 2015). J Clin Oncol. 2015;33 (18 Suppl):LBA9002. 